Kleinermann, F. De Troyer, O. Creelle, C. Pellens, B.

\title{
Adding Semantic Annotations, Navigation paths and Tour Guides to Existing Virtual Environments
}

\author{
Frederic Kleinermann ${ }^{1}$, Olga De Troyer ${ }^{1}$, Christophe Creelle ${ }^{1}$, Bram Pellens ${ }^{1}$ \\ ${ }^{1}$ Research Group WISE, Vrije Universiteit Brussel, Pleinlaan 2, 1050 Brussels, Belgium \\ \{frederic.kleinermann, olga.detroyer, christophe.creelle, bram.pellens\}@vub.ac.be
}

\begin{abstract}
Nowadays, more Virtual Environments (VEs) are becoming available on the Web. This means that VEs are becoming accessible to a larger and more diverse audience. It also means that it is more likely that the use of these VEs (i.e. how to interact with the virtual environment and the meanings of the associated virtual objects) may be different for different groups of persons. In order for a VE to be a success on the Web, end-users should easily get familiar with the VE and understand the meanings of its virtual objects. Otherwise, the end-user may be tempted to quit the VE. Therefore, annotations and the creation of navigation paths for virtual tour guides become important to ease the use of VEs. Most of the time, this is done by VR-experts and the annotations are very poor and often only text based. This paper describes an approach and associated tool that allows a layman to add or update annotations to existing VEs. In addition, annotations are not limited to text but may also be multimedia elements, i.e. images, videos, sounds. Furthermore, the approach (and the tool) also allows easy creation of navigation paths and tour guides, which can be used to adapt a VE to the needs of a user. The paper illustrates the results by means of a real case, which is a reconstruction of a coalmine site for a museum.
\end{abstract}

Keywords: Virtual Reality, Semantic Annotations, Navigation Paths, Tour guides.

\section{Introduction}

Virtual Environments (VE) are becoming more available through the Internet thanks to formats like X3D [1]. Although Virtual Reality (VR) applications are becoming visually appealing, they often lack any kind of semantics, i.e. extra, non-visual information about the Virtual Environment and its objects [2]. The success of a VR application on the Web also depends on how the user is capable of interacting with the VE and how he can add meanings to the objects inside the VE and to the VE itself. This helps him to find his way in the VE and to a certain extent adapt the VE to his needs. As a result the user will be less tempted to leave the VE. A good example of this can be found in Second Life [3] where the user is not only discovering new visually attractive virtual places, but he can start to give meanings to the different objects and places in which he is immersed. For instance, he can jump to an ancient Maya site and then find new information about a temple. 
Semantic annotation is information that is added to some media to enrich it. Semantic annotations are especially important in the context of the Semantic Web because they make the content of the Web machine-processable and enable computers and people to work in cooperation. In the same way, semantic annotations can be added to VEs and to their objects through the process of annotations. The process of annotation is not only useful for making the content machine-processable, but in the context of VE it is also very important to increase the usability of the VE (as illustrated above). In particular, this is very important in application domains such as Virtual Museums and cultural heritage where providing information is substantial.

However, the process of annotating is not easy. VEs can be annotated during development or afterwards. Currently, it is often done using authoring tools that provide very limited mechanisms for annotations. Usually, some textual annotations can be added, often even only keywords. Furthermore, the annotations are stored in the same file as the one containing the VE. VEs are often annotated by VR-experts and less often by domain experts. Another observation is that the meaning that people give to objects can change from person to person and also may depend on the task under consideration. Take for instance the meaning of an old building. For a historicist investigating that building, the annotations would be related to the research he is conducting. But for someone else it could be a story related to this building. While for an architect, the semantic annotations for that building would probably be its architectural properties.

For this reason, it is important to provide an approach where persons can easily add and update different sets of semantic annotations to existing VEs, so that existing VEs cannot only be extended with semantic annotations, but the annotations can also be customized to a particular domain, task or group of people. As a result, the same VE can be visualized in different contexts with different sets of annotations and in this way be used for different purposes.

Another important aspect also related to enhancing the usability of VEs is navigation. Navigation is very important in VEs and especially in the context of the Web or for VEs reaching a large audience. Such an audience is very demanding. They not only expect visually attractive VEs, but they also want to explore them very quickly. In general, if they need to spend too much time wondering how to interact with the VE and its objects, the chance is higher that they will leave the VE quickly. This is why navigation and virtual guided tours are very important. However, also for navigation the requirements may vary from person to person and from task to task. For instance, a guided tour inside an archeological site could be different for an audience of archeologists working on the site than for an audience of tourists visiting the site. Even for archeologists, a different guided tour may be needed to teach students than to discuss with colleagues about the findings or future excavations. Furthermore, the process of creating a navigation path and a virtual tour guide is usually not easy and therefore often performed by a VR expert. It is often not possible to easily adapt it or to create it for a particular application task or group of persons.

This paper describes an approach that helps a person (who may not be a VRexpert) to easily add and update semantic annotations to existing VEs and to easily create navigation paths and virtual tour guides that are customized towards these semantic annotations. In addition, the semantic annotations can be multimedia, i.e. 
texts, images, sounds and hyperlinks. The paper also describes the tool implemented to support the approach, and built on the top of Ajax3D [4].

The paper is structured as follows. In the next section, we provide related work. Section 3 describes the approach. Section 4 explains the tool supporting the approach and section 5 presents an example. Then, section 6 discusses the work presented in this paper. The paper ends with a conclusion and future work.

\section{Related Work}

In this section, we first review research work related to annotations and semantic for $\mathrm{VE}$, and then we review research work related to navigation and tour guides.

In [5], the notion of smart object is used to provide not only the geometric information necessary for drawing objects on screen, but also semantic information useful for manipulation purposes. The semantic information in the smart object is used by virtual characters to perform actions on/with the object, e.g., grasping, moving, operating. Using the semantic information, the user is much more aware of the sort of manipulations that he can perform. For this reason, our approach also provides these features by using the semantic annotations that have been created and customized towards a group of end-users.

In [6][7], the authors propose a method in which the domain expert is annotating the virtual world when it is being created. The world is being created using ontologies and therefore, the semantic annotation is richer. The semantic annotation can be any kind of information. The navigation can then exploit these semantic annotations using a search engine. However, the navigation assumes that the world has been created and annotated using their method.

The work described in [8] presents an annotation framework facilitating the reuse of 3D models. An extension of the MPEG-7 standard is proposed that addresses 3D content. The framework allows managing the semantic annotations of $3 \mathrm{D}$ objects. The annotations can only be specified in a textual-like format which does not make it accessible to novice users. Our approach allows adding the annotation directly inside the VE making it more user-friendly.

A similar approach as ours is given in [9] where annotations are used in order to have better communication between team members. An application is presented enabling designers to directly annotate a VE and associate the annotations with a knowledge model.

The authors in [10] have developed an approach for navigation assistance in VEs. This approach allows non-professional visitors of a VE to find their way without having previous training. To assist the user in his navigation, they give a map of the environment where landmarks are added. They provide a personal assistant that has knowledge of the VE and the current user. They also provide a navigation agent for taking the user to a particular position. Although it is a very interesting work, the information used to assist the user in his navigation is limited to the geometrical and spatial aspects of the virtual worlds and its objects. Furthermore, it is not clear how generic the approach is. 
Part of the work presented in [11] is also about navigation by querying. The author provides a querying model that allows users to find objects and scenes in virtual environment based on their size and their associated meta-information. This model is based on fuzzy logic. Nonetheless, the amount of meta-information data used in this work is still limited to object properties such as name, width, height, and locations. It is not context oriented.

In [12], the authors present a technique that allows navigation to cope with large virtual worlds. This technique uses place representation and visible landmarks that scale from town-sized to planet-sized worlds. However, the semantic annotation is only on place hierarchy, which determines which visible landmarks and place representation that users can see.

The problems generally encountered with navigation in large VEs are also acknowledged in [13]. An entertainment application is presented to educate people. Environments can be explored by means of flythrough mode or a roller coaster ride. However, the navigation paths need to be specified manually being time-consuming. Our approach enables fast creation of navigation networks directly inside the VE.

Another interesting work is given in [14] where an alternate approach is presented to associate semantic information based on the integration of X3D and the Semantic Web. The approach is illustrated with an application of a user-guide in complex environments and one of a virtual tour. A downside is that the user is not really guided in making the annotations and is therefore not very usable for a domain expert. Our approach focuses on making the annotation process more easily for domain experts or laymen.

\section{Approach}

This section explains our approach to make semantic annotations, navigation paths and virtual tour guides for existing VEs. The approach allows adding or updating semantic annotations, and to create new navigation paths and virtual tour guides directly inside the VE and in a visual way. The semantic annotations are multimedia elements, i.e. texts, videos, images and hyperlinks.

\subsection{Point of Interest (POI), Navigation Paths, Navigation landmarks}

Point of Interest. In our approach, semantic annotations are created and represented through the concept of point-of-interest (POI). POI is defined by a position, an orientation and some multimedia elements (information) can be associated with it (images, videos, hyperlinks, or texts). A single POI may contain several multimedia elements. Furthermore, a POI can also be positioned inside a complex object.

Navigation Paths and Navigation Landmarks. A navigation path is created by linking several navigation landmarks in a well-defined order. A landmark has a position and an orientation. The navigation paths will be used to guide a tour guide. 


\subsection{Designer View and End-user View}

The approach distinguishes between two views: the designer view and the end-user view. The designer view is used to create the semantic annotations, navigation paths and tour guides. In the end-user view, the annotated VE can be used, with or without using the tour guides.

Designer View. The designer view provides the designer (or domain expert) a way to add semantic annotations, to modify them or to delete them. The designer can also create navigation paths and associate an avatar to them in order to create a tour guides. We will now explain how the designer can annotate an object, create a navigation path and add a tour guide.

Semantic annotation. The designer can add a semantic annotation by positioning a POI on an object or around it. However, an object can contain (built of) several other objects. For this reason, our approach also allows annotating the objects (or parts) inside such an object. For instance, if we take an object car made of a body and four wheels, then the designer may want to annotate not only the car as a whole, but also each of its components i.e., the wheels and body.

The positioning of POIs can be done in two different ways namely using a freehand mode and using a grid mode. In the freehand mode, the designer can visually position a POI anywhere on (or inside) an object to add semantic annotations to this specific location. The designer can also define an orientation by using the orientation of his view.

As it is not always easy to position a POI using the freehand mode, the approach also provides a grid mode. In that mode, a grid of spheres overlaying the selected object is displayed. Each sphere represents a possible POI. The designer selects the spheres that he wants in order to define a POI. In other words, the grid mode has a number of predefined positions for the POIs. The grid has the size of the selected object based on its bounding box information. The number of spheres and the space between them can be customized according to the designer's specifications. The designer can also select different spheres to annotate areas of an object. For instance in the case of the coalmine site, the designer can select the object representing a building block. The building block is made of three parts namely a building, a sidewalk and a green area. By selecting a number of spheres covering the sidewalk, he can then add semantic annotations only related to the area of the sidewalk. He can repeat this for other areas.

Using POIs, the annotation process can be time-consuming as each object needs to be annotated individually. Therefore our approach also provides a way to accelerate the annotation process. After having annotated an object (either using the freehand or the grid mode), the designer can decide to propagate the semantic annotations of that object to the group of objects that have been identified to be similar. This feature is realized in our tool by using the X3D instructions like "ProtoInstance", "ExternProto", "USE", "DEF" which help to identify groups of similar objects. In other words, the semantic annotations can be created once for a complex object and be propagated to a number of similar objects populating the VE. That way, the process of annotation is less time-consuming and will also be more consistent. 
Defining a Navigation Path. To create a navigation path, a number of landmarks must be positioned. To position landmarks, the approach also uses a freehand mode and a grid mode. Similar as for defining POIs, in the freehand mode navigation landmarks are positioned visually and freely by the designer on (or inside) an object. The grid mode allows the designer to specify the navigation landmarks by selecting specific spheres of the grid. During the annotation process (see above), the designer may have added semantic annotations using the grid mode and for the same object for which the designer wants to specify a navigation path. In that case, the approach allows the designer to reuse the spheres used for the semantic annotations. For instance, suppose that the designer wants to constraint the path of the tour guide inside a coalmine tour by stating that the tour guide can only walk on the sidewalk. Suppose that the designer has annotated the building block using the grid mode. Therefore he has selected the spheres of the grid covering the building, sidewalk and green zone. When he wants to specify the navigation path for the building block, he can then do it using the semantic annotation related to sidewalk. As a result the navigation path for that object will be constrained to the volume defined by the selected spheres for the semantic annotation of the sidewalk.

A navigation path is composed by linking different landmarks in a well-defined order. When the path in followed (in the end-user view), the transition from one landmark to another one will be done by means of the shortest path.

Furthermore, the designer can also confirm to propagate the navigation path defined on an object to a group of similar objects. Like in the process of adding semantic annotations, this may accelerates the process of defining navigation paths.

Defining a Tour Guide. To define a tour guide, an avatar must be associated with a navigation path. In our approach, the tour guide (avatar) will move according to the navigation path associated to it and will show the semantic information (annotations) associated to a POI when it encounters a POI in his range. The range can be specified and corresponds to the radius of a sphere where the center of the sphere is the current position of the avatar at a given time.

End-user view. This view allows an end-user to select a tour guide for a VE. Furthermore, the end-user has the possibility to directly go to a POI, by jumping from where he is in the VE to a POI. The selection of a POI is based on a match between search information entered by the end-user and the semantic annotations associated with POIs. In the current implementation, the matching is only done for text.

\section{Software Architecture}

To support the approach, we opted for a Web tool that can be accessed using a classical browser and can be used by a large audience. To achieve this, we have implement it using Ajax3D [4], which combines AJAX with X3D through the Flux player and its Scene Authoring Interface [4]. The tool consists of three modules, namely a server and two different types of clients. Figure 1 shows the architecture. 


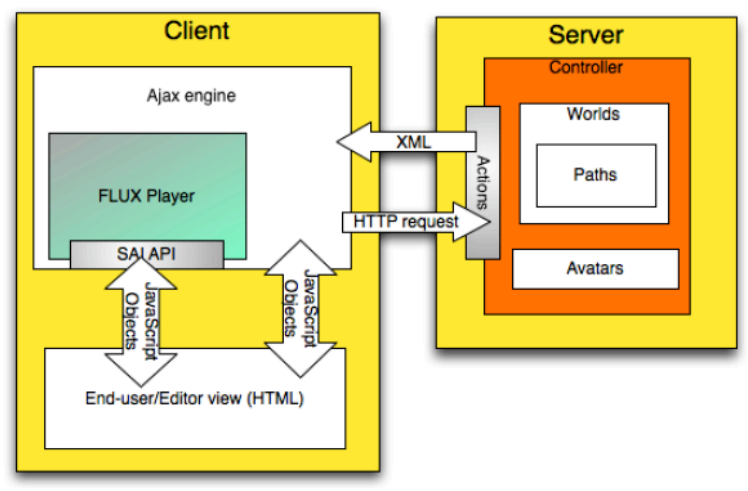

Fig. 1. Software Architecture

\subsection{Server}

The server is implemented as a Java servlet running on Apache tomcat. The server is responsible to store VEs, avatars, semantic annotations and navigation paths. The server is accessed through HTTP requests and XML and X3D files are sent from the Server to the Client. Furthermore, with AJAX it is also possible to send XML files from the Client to the Server. That way, semantic annotations can be added and updated, new navigation paths and virtual tour guides can be created. All this information is stored at the server. This information can then be accessed by the enduser to view the VE with its virtual tour guides and semantic annotations.

\subsection{Clients}

The tool has two different types of clients namely a Designer View Client and an Enduser View Client. Both clients use AJAX to send requests to the server and retrieve X3D files and XML files containing a virtual tour guide and semantic annotations. Communication with the X3D player is performed through the SAI supported by Flux Player [4]. Both clients run in a Web browser.

Designer View Client. The designer, running the Designer View Client in his Web browser, can select a VE from a list of VEs that have been uploaded on the server. Then, he can add semantic annotations, create navigation paths and tour guides. Note that currently our tool only supports human avatars based on H-Anim [15].

End-user View Client. The End-user View Client allows an end-user to select a VE and view it. He can select and follow a tour guide that has been created for this VE or walk around freely and see the different semantic annotations once he encounters a POI. Furthermore, when the tour guide encounters a POI, the view of the end-user can change according to the camera viewpoint specified by the designer, so that the enduser can see the object from a particular angle. He can also see the semantic annotations associated to that particular POI. 


\section{Example}

The described approach has been applied in the cultural heritage context on a museum application consisting of a reconstruction of the Beeringen Coalmine ${ }^{1}$ site [16]. The virtual coalmine site itself has been constructed using another tool called OntoWorld [6][7]. Here, we have used our tool to add semantic annotations and to create a navigation path and a virtual tour guide for this particular VE.

By using the Designer View Client, the designer can add semantic annotations by using either the freehand mode or the grid mode. Figure 2 shows the screen after the user having positioned (through the freehand mode) a POI and attaching some semantic annotation (here some text, images and videos). In our tool, in the freehand mode, POIs are represented by red cones. Remember that the designer can also create a navigation path by positioning navigation landmarks. Figure 3 shows an example of the designer positioning navigation landmarks represented by yellow cones in the freehand mode. Figure 4 shows the designer creating a navigation path for a particular building using the grid mode.

Once the navigation path has been stored, the designer can create a tour guide by selecting an avatar and a navigation path. In this example, a camera is used as avatar.

Then, an end-user can select the VE and the tour guide through his Web browser by loading the End-user View Client. Figure 5 shows the screen after an end-user having selected the virtual coalmine site with a tour guide. We see how the different semantic annotations are showed once the tour guide encounters POIs on his path. In figure 5, the tour guide encounters a POI. Its related semantic annotations are then displayed (here these are images, videos and texts). The images and videos are represented by thumbnails (see left side of figure 5 in the panel called "Tour Guide") and can be clicked by the end-user to view them.

${ }^{1}$ An old coalmine site in Belgium 


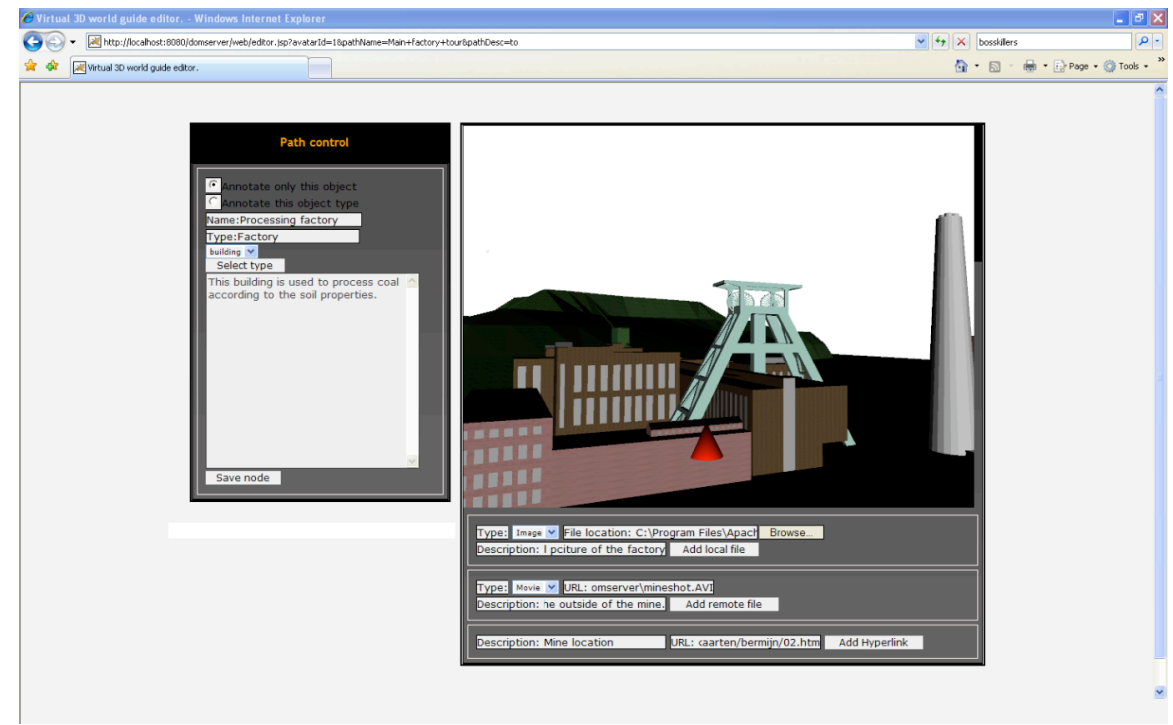

Fig. 2. Designer View Client. The designer is adding a POI (red cone) to one building of the main coalmine site using the freehand mode.

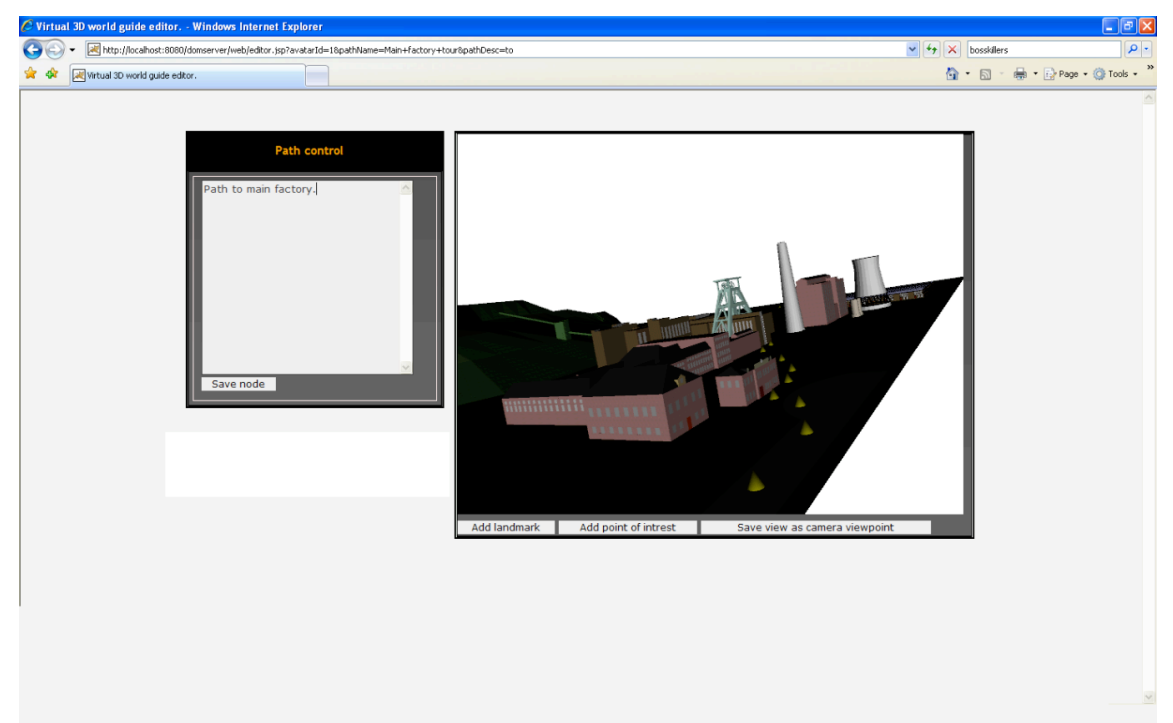

Fig. 3. Designer View Client. The designer is adding landmarks (yellow cones) to create a path around the coalmine site using the freehand mode. 


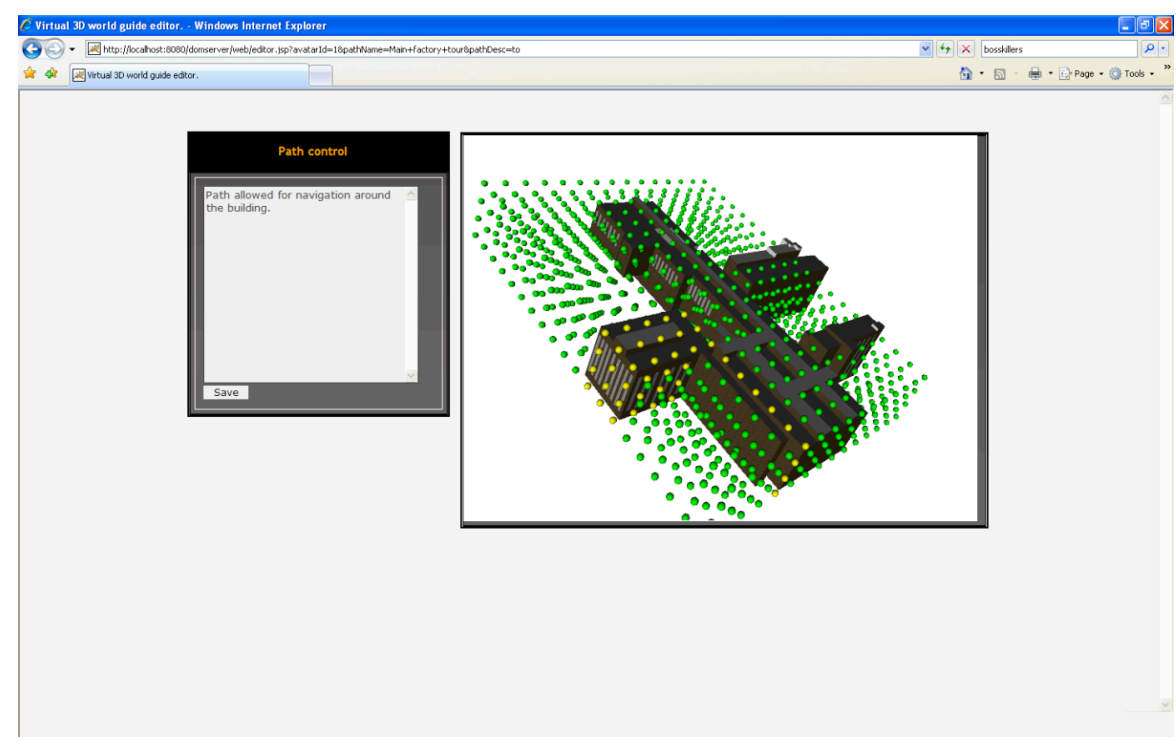

Fig. 4. Designer View Client. The designer is adding landmarks to create a path around a building of the coalmine site using the grid mode.

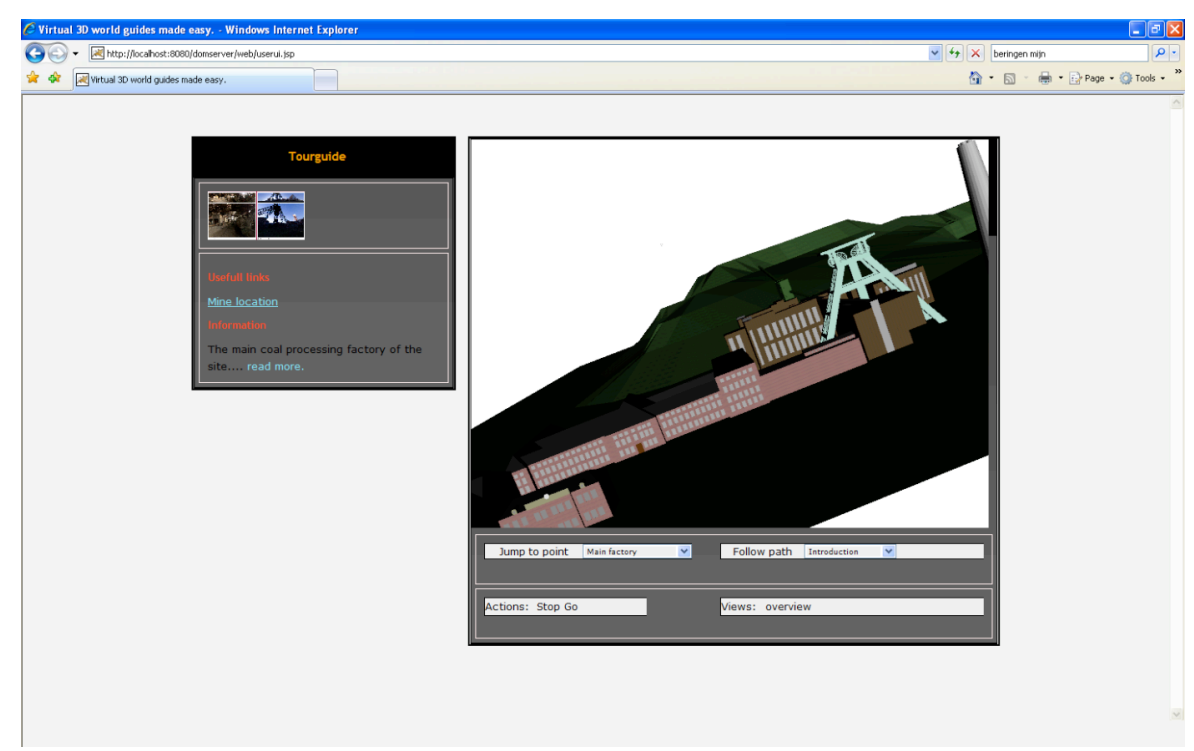

Fig. 5. End-user View Client. The user is following a tour guide of the coalmine site. 


\section{Discussion}

The approach proposed provides an easy way to add and modify semantic annotations to any VE, and to create navigation paths and virtual tour guides. Different multimedia types of semantic annotations are possible, i.e. images, text, videos and hyperlinks. The approach also provides a way to make the annotation process less time-consuming by means of a propagation mechanism and to create navigation paths for virtual objects composed of several objects. To add annotations and to specify landmarks two different modes are available. The freehand mode allows free positioning, while the grid mode uses predefined grids of spheres. In addition, the concept of grid can be combined with the semantic annotations. To ease the annotation process further, we consider adding the possibility to allow the designer to position POIs or navigation landmarks by means of so-called spatial relations. For instance, the designer could specify that a POI should be positioned left of the Mayan temple at a certain distance. This could also be used for positioning navigation landmarks.

This approach and the tool should allow designers to quickly customize VEs with semantic annotations and navigation paths. A VE can have multiple sets of semantic annotations, navigation paths and tour guides. Each of them can be used to represents a particular user view or to support a particular task. The tool implementing this approach is not limited to a certain type of VE but is very general. It uses X3D and Ajax $3 \mathrm{D}$ and therefore the tool can be deployed on the Web and is accessible to a large audience.

Note that the tool can also be used to create virtual "post-its". A person can quickly add some annotations and a tour guide and then share this with other users. This can be very useful as a communication tool in education, or for archeologists or engineers to relate different types of annotations (such as comments, marks, errors, ...) to a particular virtual model and then share them with other stakeholders (students, colleagues, ...) who can then read the annotations and add their own.

\section{Conclusions and Future Work}

This paper has presented an approach that allows people who may not be VR-experts, to easily add semantic annotations (in the form of multimedia information) to existing VEs and to create tour guides by easily defining navigation paths in these VEs. A tool has been developed implementing this approach. The tool is based on Ajax3D.

Future work will focus on adding semantic annotation to behaviors and to allow creating navigation paths more intuitively using semantic annotations and spatial relations. Furthermore, some user experiments need to be conducted where the tools are used and tested, hereby investigating the intuitiveness and flexibility of our approach.

Acknowledgments.

This research was carried out in context of the VR-DeMo project which is directly funded by the IWT, a Flemish subsidy organization from Belgium (IWT 030248). 


\section{References}

1. Brutzman, D., Daly, L., X3D: Extensible 3D Graphics for Web Authors. Morgan Kaufmann (2007)

2. Martinez, J., Delgado Mata, C.: A Basic Semantic Common Level for Virtual Environments. Int. Journal. of Virtual Reality, Vol. 5. No. 3. (2006) 25 - 32

3. Rymaszewski, M., Wagner, J., Wallace, M., Winters, C., Ondrejka, C., BatstoneCunningham, B.: Second Life, The official Guide, Sybex (2007)

4. Parisi, T. : Ajax3D : The Open Platform for Rich 3D Web Applications. Whitepaper. Media Machines, Inc. (2006)

5. Abaci, T., Mortara, M., Patane, G., Spagnuolo, M., Vexo, F., Thalmann, D.: Bridging Geometry and Semantics for Object manipulation and Grasping. In: The Workshop towards Semantic Virtual Environment (SVE), Villars, Switzerland (2005) 110-119

6. Kleinermann, F., De Troyer, O., Mansouri, H., Romero, R., Pellens, B., Bille, W.: Designing Semantic Virtual Reality Applications. In: Proceedings of the 2nd INTUITION International Workshop, Senlis, France (2005) 5-10

7. De Troyer, O., Kleinermann, F., Mansouri, H., Pellens, B., Bille, W., Fomenko, V.: Developping semantic VR-shops for e-Commerce, In: (eds.) Magoulas, G.D., Lepouras, G., Vassilakis, C.: Special Issue of VIRTUAL REALITY: "Virtual Reality in the e-Society". Springer-Verlag, SpringerLink London (2006) 20-60

8. Bilasco, I.M., Gensel, J., Villanova-Oliver, M., Martin, H.: An MPEG-7 framework enhancing the reuse of 3D models. In: Proceedings of the international conference on 3D web technology, Columbia, USA (2006) $65-74$

9. Aubry, S., Thouvenin, I., Lenne, D., Okawa, S.: Knowledge integration for annotating in virtual environments. Int. J. Product and Development. Vol. 4, No. 6. Inderscience Publishing (2007) 533-546

10. Van Dijk, B., Op den Akker, R., Nijholt, A., Zwiers, J.: Navigation Assistance in Virtual Worlds. Special Series on Community Informatics. Vol. 6. Information Science Journal (2003) 115-124

11. Ibanez, J.: An intelligent Guide for Virtual Environments With Fuzzy Queries and Flexible Management of Stories. PhD thesis. Department of Computer Science, University of Murcia, Spain (2004)

12. Pierce, J.S., Pausch, R.: Navigation with place representations and visible landmarks. In: Proceedings of the IEEE Virtual Reality Conference, Chicago, USA (2004) 173-288

13. Olanda R., Pérez, M., Morillo, P., Fernández, M., Casas, S.: Entertainment virtual reality system for simulation of spaceflights over the surface of the planet Mars. In: Proceedings of the ACM symposium on Virtual reality software and technology. Limassol, Cyprus (2006) $123-132$

14. Pittarello, F., De Faveri, A.: Semantic description of 3D environments: a proposal based on web standards. In: Proceedings of the international conference on 3D web technology, Columbia, USA (2006) 85 - 95

15. The Web3D Consortium, H-Anim 2001 - Humanoid Animation Specification, http://www.h-anim.org/Specifications/H-Anim200x

16. --, Beringen-Mijn: http://www.toerismeberingen.be/html/mijnmuseum. Accessed 10th April $\underline{2007}$ 